\title{
Further results on existence of positive solutions of generalized fractional boundary value problems
}

\author{
Hojjat Afshari ${ }^{*}$, Mohammed S. Abdo² and Jehad Alzabut ${ }^{3}$ (D)
}

\section{"Correspondence:}

hojat.afshari@yahoo.com

'Department of Mathematics,

Faculty of Basic Science, University

of Bonab, Bonab, Iran

Full list of author information is

available at the end of the article

\section{Springer}

\begin{abstract}
This paper studies two classes of boundary value problems within the generalized Caputo fractional operators. By applying the fixed point result of $\alpha-\phi$-Geraghty contractive type mappings, we derive new results on the existence and uniqueness of the proposed problems. Illustrative examples are constructed to demonstrate the advantage of our results. The theorems reported not only provide a new approach but also generalize existing results in the literature.
\end{abstract}

Keywords: Generalized Caputo differential equation; $\alpha-\phi$-Geraghty contractive; Positive solution

\section{Introduction}

Recently, it has been realized that fractional calculus (FC) has played a very important role in different areas of research; see $[26,32]$ and the references cited therein. Consequently, fractional differential equations (FDEs) have grasped the interest of many researchers working in diverse applications [22, 39]. Most relevant results have been obtained in terms of the classical fractional derivatives (FDs) of Riemann-Liouville (RL), Caputo (Ca), Katugampola (Ka), Hadamard (Ha), Hilfer (Hi) FDs etc.

Generalized fractional derivatives (GFDs) with respect to another function $\kappa$ have been considered in $[32,41]$ as a generalization of RL fractional operator (FO). The GFD is different from the other classical FD because the kernel appears in terms of another function $\kappa$. Recently, Almeida in [13] presented a generalized version of Ca with some advantageous properties. Many properties of the generalized FO can be found in [11, 32, 33, 41, 45]. The advantage of studying the generalized FD lay in providing a general platform that includes all particular derivatives. For some special cases of a function $\kappa$, one can realize that $\kappa$ Ca FD can be reduced to the (Ca, when $\kappa(t) \rightarrow t$ see [32], Ca-Ha, when $\kappa(t) \rightarrow \log t$ [28], Ca-Ka, when $\left.\kappa(t) \rightarrow t^{\rho}(\rho>0)[30,31]\right)$ FD.

On the other hand, the investigation of existence and uniqueness of solutions to several types of fractional (impulsive, functional, evolution, etc.) differential equations is the main topic of applied mathematics research. Many interesting results with regard the existence,

(c) The Author(s) 2020. This article is licensed under a Creative Commons Attribution 4.0 International License, which permits use sharing, adaptation, distribution and reproduction in any medium or format, as long as you give appropriate credit to the original author(s) and the source, provide a link to the Creative Commons licence, and indicate if changes were made. The images or other third party material in this article are included in the article's Creative Commons licence, unless indicated otherwise in a credit line to the material. If material is not included in the article's Creative Commons licence and your intended use is not permitted by statutory regulation or exceeds the permitted use, you will need to obtain permission directly from the copyright holder. To view a copy of this licence, visit http://creativecommons.org/licenses/by/4.0/. 
uniqueness, and stability of solutions or positive solutions by using some fixed point (FP) theorem have been discussed in Refs. [1, 12, 18, 19, 21, 23, 24, 27, 35, 37, 38, 44].

For the purpose of completeness, we refer thereafter to some relevant papers that deal with the existence of positive solutions involving classical $\mathrm{Ca}$ and $\mathrm{RL}$ derivatives. More precisely, the authors in [17] studied the existence and multiplicity of positive solutions for the following problem:

$$
\left\{\begin{array}{l}
{ }^{\mathrm{RL}} D_{0+}^{l} \varpi(\varkappa)+g(\varkappa, \varpi(\varkappa))=0, \quad \varkappa \in(0,1), \\
\varpi(0)=\varpi(1)=0
\end{array}\right.
$$

where $1<\imath \leq 2$, and ${ }^{\mathrm{RL}} D_{0_{+}}^{l}$ is RL FO. Also, the problem

$$
\left\{\begin{array}{l}
{ }^{C} D_{0+}^{\iota} \varpi(\varkappa)+g(\varkappa, \varpi(\varkappa))=0, \quad \varkappa \in(0,1), \\
\varpi(0)+\varpi^{\prime}(0)=0, \quad \varpi(1)+\varpi^{\prime}(1)=0,
\end{array}\right.
$$

was discussed in [48], where $1<\imath \leq 2$, and ${ }^{C} D_{0_{+}}^{\imath}$ is the CF operator.

For some recent findings on GFDs with respect to another function $\kappa$, see $[2,3,14,15$, $25,34,36,42,43,46,47]$.

In other direction, Karapinar and Samet introduced the notion of generalized $\alpha-\psi$ Geraghty contractive ( $\alpha-\psi-\mathrm{GC})$ type mappings (see [29]). The generalized $\alpha-\psi-\mathrm{GC}$ in complete b-metric spaces (b-MS) and their applications in b-metric spaces b-MS was introduced in $[4-10,16,40]$.

To the best of our observation, the investigation of positive solutions to fractional BVP has not been studied within $\kappa$-Ca and $\kappa$-RL FOs yet. Moreover, the FP technique based on $\alpha-\psi$-GC has never been applied to such problems.

Inspired by the above results and motivated by the recent evolutions in $\kappa$-fractional calculus, in this paper, we apply the FP technique of $\alpha-\psi$-GC type mappings to investigate the existence of positive solutions for the following fractional BVPs:

$$
\left\{\begin{array}{l}
{ }^{C} D_{0+}^{\imath, \kappa} \varpi(\varkappa)+g(\varkappa, \varpi(\varkappa))=0, \quad \varkappa \in(0,1), \\
\varpi(0)=\varpi(1)=0,
\end{array}\right.
$$

and

$$
\left\{\begin{array}{l}
{ }^{C} D_{0+}^{\imath, \kappa} \varpi(\varkappa)+g(\varkappa, \varpi(\varkappa))=0, \quad \varkappa \in(0,1), \\
\varpi(0)+\varpi^{\prime}(0)=0, \quad \varpi(1)+\varpi^{\prime}(1)=0 .
\end{array}\right.
$$

where $1<\imath \leq 2$, and ${ }^{C} D_{0+}^{t, \kappa}$ is $\kappa$-FD of order $\imath$ in the sense the $\kappa$-Ca operator, and $g: \digamma \times$ $\mathbb{R} \rightarrow \mathbb{R}^{+}$is a continuous function. Throughout the article $\digamma=[0,1]$ and $\mathbb{R}^{+}=[0, \infty)$.

We claim that our approach is new and the reported results are different form existing ones in the literature.

The remaining parts of the paper are outlined as follows: Some preliminary facts needed for the proofs of the main results are recalled in Sect. 2. In Sect. 3, we prove the existence of positive solutions for problems (1) and (2) by the aid of the FP result of $\alpha-\psi$-GC type mappings. Examples are given in Sect. 4 to check the applicability of the theoretical findings. We end the paper by a conclusion. 


\section{Preliminaries}

Definition 2.1 ([32]) Let $\iota>0$ and $\kappa$ be an increasing function, having a continuous derivative $\kappa^{\prime}$ on $(a, b)$. The left-sided $\kappa$-RL fractional integral of a function $h$ with respect to $\kappa$ on $[a, b]$ is defined by

$$
I_{a^{+}}^{\iota, \kappa} h(\varrho)=\frac{1}{\Gamma(\iota)} \int_{a}^{\varrho} \kappa^{\prime}(\varsigma)[\kappa(\varrho)-\kappa(\varsigma)]^{\iota-1} h(\varsigma) d \varsigma, \quad \varrho>a, \iota>0,
$$

provided that $I_{a^{+}}^{l, \kappa}$ exists. Note that when $\kappa(\varrho)=\varrho$, we obtain the known classical RL fractional integral.

Definition 2.2 $([32,41])$ Let $\iota>0, n$ be the smallest integer greater than or equal to $\iota$ and $h \in L^{p}[a, b], p \geq 1$ let $\kappa \in C^{n}[a, b]$ an increasing function such that $\kappa^{\prime}(\varrho) \neq 0$, for all $\varrho \in[a, b]$. The left-sided $\kappa$-RL FD of $h$ of order $\iota$ is given by

$$
D_{a^{+}}^{l ; \kappa} h(\varrho)=\left(\frac{1}{\kappa^{\prime}(\varrho)} \frac{d}{d \varrho}\right)^{n} I_{a^{+}}^{n-\iota, \kappa} h(\varrho), \quad n-1<\iota<n, n \in \mathbb{N} .
$$

Definition $2.3([13,14])$ Let $n-1<\iota<n, h \in C^{n}[a, b]$, and let $\kappa \in C^{n}[a, b]$ an increasing function such that $\kappa^{\prime}(\varrho) \neq 0$, for all $\varrho \in[a, b]$. The left-sided $\kappa$-Ca FD of $h$ of order $\iota$ is given by

$$
{ }^{C} D_{a^{+}}^{\ell ; \kappa} h(\varrho)=I_{a^{+}}^{n-l, \kappa} D^{n, \kappa} h(\varrho)
$$

where $D^{n, \kappa}:=\left(\frac{1}{\kappa^{\prime}(\varrho)} \frac{d}{d \varrho}\right)^{n}$, and $n=[\iota]+1$.

Definition 2.4 ([20]) Let $M$ be a nonempty set and $r \geq 1$. A mapping $d: M \times M \rightarrow \mathbb{R}^{+}$is said to be a $b$-metric if for $\varrho, \varsigma, \varpi \in M$;

$\left(b M_{1}\right) d(\varrho, \varsigma)=0$ if and only if $\varsigma=\varrho$;

$\left(b M_{2}\right) d(\varrho, \varsigma)=d(\varsigma, \varrho)$;

$\left(b M_{3}\right) d(\varrho, \varpi) \leq r[d(\varrho, \varsigma)+d(\varsigma, \varpi)]$.

The pair $(M, d)$ is called a b-MS with constant $r$.

Let $\Phi$ be set of all increasing and continuous functions $\phi: \mathbb{R}^{+} \rightarrow \mathbb{R}^{+}$satisfying the property: $\phi(c \varrho) \leq c \phi(\varrho) \leq c \varrho$ for $c>1$ and $\phi(0)=0$. We denote by $\mathcal{F}$ the family of all nondecreasing functions $\lambda: \mathbb{R}^{+} \rightarrow\left[0, \frac{1}{r^{2}}\right)$ for some $r \geq 1$.

Definition 2.5 ([8]) Let $(M, d)$ be a b-MS and $T: M \rightarrow M$, we say that $T$ is a generalized $\alpha$ - $\phi$-GC type mapping whenever there exists $\alpha: M \times M \rightarrow \mathbb{R}^{+}$such that

$$
\alpha(\varrho, \varsigma) \phi\left(r^{3} d(T \varrho, T \varsigma)\right) \leq \lambda(\phi(d(\varrho, \varsigma))) \phi(d(\varrho, \varsigma))
$$

for $\varrho, \varsigma \in M$, where $\lambda \in \mathcal{F}$ and $\phi \in \Phi$.

Definition 2.6 ([40]) For $M(\neq \emptyset)$, let $T: M \rightarrow M$ and $\alpha: M \times M \rightarrow \mathbb{R}^{+}$be given mappings. We say that $T$ is $\alpha$-admissible if, for $\varrho, \varsigma \in M$, we have

$$
\alpha(\varrho, \varsigma) \geq 1 \quad \Longrightarrow \quad \alpha(T \varrho, T \varsigma) \geq 1
$$


Theorem 2.7 ([8]) Let $(M, d)$ be a complete $b$-MS and $T: M \rightarrow M$ be a generalized $\alpha-\phi$ GC type mapping such that

(i) $T$ is $\alpha$-admissible;

(ii) there exists $\varrho_{0} \in M$ such that $\alpha\left(\varrho_{0}, T \varrho_{0}\right) \geq 1$;

(iii) if $\left\{\varrho_{n}\right\} \subseteq M$ with $\varrho_{n} \rightarrow \varrho$ and $\alpha\left(\varrho_{n}, \varrho_{n+1}\right) \geq 1$, then $\alpha\left(\varrho_{n}, \varrho\right) \geq 1$.

Then $T$ has a FP.

Lemma 2.8 ([46]) Let $g \in C(\digamma)$ and $1<\iota \leq 2$. Then the FBVP

$$
\left\{\begin{array}{l}
{ }^{C} D_{a+}^{\iota, \kappa} \varpi(\varkappa)+g(\varkappa, \varpi(\varkappa))=0, \quad \varkappa \in(0,1), \\
\varpi(0)=0, \quad \varpi(1)=0,
\end{array}\right.
$$

is equivalent to

$$
\varpi(\varkappa)=\int_{0}^{1} \mathcal{G}(\varkappa, \vartheta) \kappa^{\prime}(\vartheta) g(\vartheta, \varpi(\vartheta)) d \vartheta,
$$

where

$$
\mathcal{G}(\varkappa, \vartheta)=\frac{(\kappa(\varkappa)-\kappa(0))^{\iota-1}}{(\kappa(1)-\kappa(0))^{\iota-1} \Gamma(\iota)}\left\{\begin{array}{c}
(\kappa(1)-\kappa(\vartheta))^{\iota-1}-\frac{(\kappa(1)-\kappa(0))^{\iota-1}}{(\kappa(\varkappa)-\kappa(0))^{\iota-1}}(\kappa(\varkappa)-\kappa(\vartheta))^{\iota-1}, \\
0 \leq \vartheta \leq \varkappa \leq 1, \\
(\kappa(1)-\kappa(\vartheta))^{\iota-1}, \\
0 \leq \varkappa \leq \vartheta \leq 1 .
\end{array}\right.
$$

Lemma 2.9 ([46]) For the function $\mathcal{G}$ defined by (5) we have;

(i) $\mathcal{G}(\varkappa, \vartheta)>0$, for all $\varkappa, \vartheta \in(0,1)$.

(ii) For $\vartheta \in(0,1)$, there exists a positive function $\gamma$ such that

$$
\min _{\varkappa \in[1 / 4,3 / 4]} \mathcal{G}(\varkappa, \vartheta) \geq \gamma(\vartheta) \max _{\varkappa \in \digamma} \mathcal{G}(\varkappa, \vartheta)
$$

Lemma 2.10 ([46]) Let $g \in C[a, b]$, and $1<\iota \leq 2$, then the FBVP

$$
\begin{cases}{ }^{C} D_{0+}^{\ell, \kappa} \varpi(\varkappa)-g(\varkappa)=0, & \varkappa \in(0,1), \\ \varpi(0)+\varpi^{\prime}(0)=0, & \varpi(1)+\varpi^{\prime}(1)=0,\end{cases}
$$

has a solution

$$
\varpi(\varkappa)=\int_{0}^{1} \Lambda(\varkappa, \vartheta) \kappa^{\prime}(\vartheta) g(\vartheta) d \vartheta,
$$

where

$$
\begin{aligned}
\Lambda(\varkappa, \vartheta)=( & \left.\Gamma(\iota-1)\left[(\kappa(1)-\kappa(0))+\left(\kappa^{\prime}(1)-\kappa^{\prime}(0)\right)\right]\right)^{-1} \\
& \times\left\{\begin{array}{c}
\left(( \kappa ^ { \prime } ( 0 ) + \kappa ( 0 ) - \kappa ( \varkappa ) ) \left[\left((\kappa(1)-\kappa(\vartheta))^{\iota-2}+\frac{1}{\iota-1}(\kappa(1)-\kappa(\vartheta))^{\iota-1}\right]\right.\right. \\
+\frac{(\kappa(1)-\kappa(0))+\left(\kappa^{\prime}(1)-\kappa^{\prime}(0)\right)}{\iota-1}(\kappa(\varkappa)-\kappa(\vartheta))^{\iota-1}, \quad 0 \leq \vartheta \leq \varkappa \leq 1, \\
\left(( \kappa ^ { \prime } ( 0 ) + \kappa ( 0 ) - \kappa ( \varkappa ) ) \left[\left((\kappa(1)-\kappa(\vartheta))^{\iota-2}+\frac{1}{\iota-1}(\kappa(1)-\kappa(\vartheta))^{\iota-1}\right],\right.\right. \\
0 \leq \varkappa \leq \vartheta \leq 1 .
\end{array}\right.
\end{aligned}
$$


Lemma 2.11 ([46]) Let $\kappa(\varkappa) \leq \kappa(0)+\kappa^{\prime}(0)$, then (7) satisfies $\Lambda(\varkappa, \vartheta)>0$, for all $\vartheta, \varkappa \in$ $(0,1)$. Besides, there exists a positive function $v \in(0,1)$, such that

$$
\min _{\varkappa \in[1 / 4,3 / 4]} \Lambda(\varkappa, \vartheta) \geq v(\vartheta) M(\vartheta), \quad \vartheta \in(0,1)
$$

and

$$
\max _{\varkappa \in \digamma} \Lambda(\varkappa, \vartheta) \leq M(\vartheta)
$$

where

$$
\begin{aligned}
& m(\vartheta)=\left(\kappa^{\prime}(0)+\kappa(0)-\kappa(3 / 4)\right)\left[(\kappa(1)-\kappa(\vartheta))^{\iota-2}+\frac{1}{\iota-1}(\kappa(1)-\kappa(\vartheta))^{\iota-1}\right], \\
& M(\vartheta)=\left(\kappa(1)+\kappa^{\prime}(1)\right)\left[(\kappa(1)-\kappa(\vartheta))^{\iota-2}+\frac{2}{\iota-1}\left((\kappa(1)-\kappa(\vartheta))^{\iota-1}\right],\right.
\end{aligned}
$$

and

$$
\begin{aligned}
v(\vartheta) & =\frac{m(\vartheta)}{M(\vartheta)}=\frac{\kappa^{\prime}(0)+\kappa(0)-\kappa(3 / 4)}{\kappa^{\prime}(1)+\kappa(1)} \frac{(\iota-1)(\kappa(1)-\kappa(\vartheta))^{l-2}+(\kappa(1)-\kappa(\vartheta))^{\iota-1}}{(\iota-1)(\kappa(1)-\kappa(\vartheta))^{\iota-2}+2(\kappa(1)-\kappa(\vartheta))^{\iota-1}}, \\
\vartheta & \in(0,1) .
\end{aligned}
$$

\section{Main results}

Let $M=C\left(\digamma, \mathbb{R}^{+}\right)$and $d: M \times M \rightarrow \mathbb{R}^{+}$be given by

$$
d(\varpi, w)=\left\|(\varpi-w)^{2}\right\|_{\infty}=\sup _{\vartheta \in[0,1]}(\varpi(\vartheta)-w(\vartheta))^{2} .
$$

Then, $(M, d)$ is a complete b-MS with $r=2$.

\section{Theorem 3.1 Suppose that}

(i) $\exists g: \digamma \times \mathbb{R}^{+} \rightarrow \mathbb{R}^{+}$such that

$$
\begin{aligned}
& |g(\vartheta, \varpi(\vartheta))-g(\vartheta, w(\vartheta))| \\
& \quad \leq \frac{1}{2 \sqrt{2}} \frac{\Gamma(\iota+1)}{(\kappa(1)-\kappa(0))^{\iota}} \sqrt{\phi\left(\left\|(\varpi-w)^{2}\right\|_{\infty}\right) \lambda\left(\phi\left(\left\|(\varpi-w)^{2}\right\|_{\infty}\right)\right)},
\end{aligned}
$$

where $\phi \in \Phi$ and $\lambda \in \mathcal{F}$;

(ii) $\exists \varpi_{0} \in C(\digamma)$ and $\mu: \mathbb{R}^{2} \rightarrow \mathbb{R}$ with $\mu\left(\varpi_{0}(\vartheta), \int_{0}^{1} \mathcal{G}(\varkappa, \vartheta) \kappa^{\prime}(\vartheta) g\left(\vartheta, \varpi_{0}(\vartheta)\right) d \vartheta\right) \geq 0$, $\vartheta \in \digamma$;

(iii) for $\vartheta \in \digamma$ and $\varpi, w \in C(\digamma), \mu(\varpi(\vartheta), w(\vartheta)) \geq 0$ implies

$$
\mu\left(\int_{0}^{1} \mathcal{G}(\varkappa, \vartheta) \kappa^{\prime}(\vartheta) g(\vartheta, \varpi(\vartheta)) d \vartheta, \int_{0}^{1} \mathcal{G}(\varkappa, \vartheta) \kappa^{\prime}(\vartheta) g(\vartheta, w(\vartheta)) d \vartheta\right) \geq 0
$$

(iv) If $\left\{\varpi_{n}\right\} \subseteq C(\digamma)$ with $\varpi_{n} \rightarrow \varpi$ and $\mu\left(\varpi_{n}, \varpi_{n+1}\right) \geq 0$, then $\mu\left(\varpi_{n}, \varpi\right) \geq 0$.

Then the problem (4) has at least one solution. 
Proof By Lemma 2.8, $\varpi \in C(\digamma)$ is a solution of (6) if and only if $\varpi$ is a solution of the integral equation $\varpi(\varkappa)=\int_{0}^{1} \mathcal{G}(\varkappa, \vartheta) \kappa^{\prime}(\vartheta) g(\vartheta, \varpi(\vartheta)) d \vartheta, \varkappa \in \digamma$. Define, $O: C(\digamma) \rightarrow C(\digamma)$ by $O \varpi(\varkappa)=\int_{0}^{1} \mathcal{G}(\varkappa, \vartheta) \kappa^{\prime}(\vartheta) g(\vartheta, \varpi(\vartheta)) d \vartheta$. We find a FP of $O$. Now, let $\varpi, w \in C(\digamma)$ be such that $\mu(\varpi(\varkappa), w(\varkappa)) \geq 0$. On one hand we have

$$
\begin{aligned}
\mid O \varpi(\varkappa)-O w(\varkappa))\left.\right|^{2} \\
\quad=\left|\int_{0}^{1} \mathcal{G}(\varkappa, \vartheta) \kappa^{\prime}(\vartheta) g(\vartheta, \varpi(\vartheta)) d \vartheta-\int_{0}^{1} \mathcal{G}(\varkappa, \vartheta) \kappa^{\prime}(\vartheta) g(\vartheta, w(\vartheta)) d \vartheta\right|^{2} \\
\quad \leq\left[\int_{0}^{1} \mathcal{G}(\varkappa, \vartheta) \kappa^{\prime}(\vartheta)|g(\vartheta, \varpi(\vartheta))-g(\vartheta, w(\vartheta))| d \vartheta\right]^{2} .
\end{aligned}
$$

By Lemma 2.8, for $0<\varkappa<\vartheta<1$ we have

$$
\begin{aligned}
\int_{0}^{1} \mathcal{G}(\varkappa, \vartheta) \kappa^{\prime}(\vartheta) d \vartheta & =\frac{(\kappa(\varkappa)-\kappa(0))^{\iota-1}}{(\kappa(1)-\kappa(0))^{\iota-1} \Gamma(\iota)} \int_{0}^{1}(\kappa(1)-\kappa(\vartheta))^{\iota-1} \kappa^{\prime}(\vartheta) d \vartheta \\
& =\frac{(\kappa(\varkappa)-\kappa(0))^{\iota-1}}{(\kappa(1)-\kappa(0))^{\iota-1} \Gamma(\iota)}\left[\frac{-(\kappa(1)-\kappa(\vartheta))^{\iota}}{\iota}\right]_{0}^{1} \\
& \leq \frac{(\kappa(1)-\kappa(0))^{\iota-1}}{(\kappa(1)-\kappa(0))^{\iota-1} \Gamma(\iota+1)}(\kappa(1)-\kappa(0))^{\iota} \\
& =\frac{(\kappa(1)-\kappa(0))^{\iota}}{\Gamma(\iota+1)} .
\end{aligned}
$$

For $0<\vartheta<\varkappa<1$, the same estimates can be proved in analogous way to the previous one. So we will omit it.

Using (i), we get

$$
\begin{array}{rl}
\int_{0}^{1} & \mathcal{G}(\varkappa, \vartheta) \kappa^{\prime}(\vartheta)|g(\vartheta, \varpi(\vartheta))-g(\vartheta, w(\vartheta))| d \vartheta \\
\quad \leq & \frac{(\kappa(1)-\kappa(0))^{\iota}}{\Gamma(\iota+1)}|g(\vartheta, \varpi(\vartheta))-g(\vartheta, w(\vartheta))| \\
\quad \leq \frac{1}{2 \sqrt{2}} \sqrt{\phi\left(\left\|(\varpi-w)^{2}\right\|_{\infty}\right) \lambda\left(\phi\left(\left\|(\varpi-w)^{2}\right\|_{\infty}\right)\right)} .
\end{array}
$$

Thus,

$$
\mid O \varpi(\varkappa)-O w(\varkappa))\left.\right|^{2} \leq \frac{1}{8} \phi\left(\left\|(\varpi-w)^{2}\right\|_{\infty}\right) \lambda\left(\phi\left(\left\|(\varpi-w)^{2}\right\|_{\infty}\right)\right) .
$$

Put $\alpha: C(\digamma) \times C(\digamma) \rightarrow \mathbb{R}^{+}$by

$$
\alpha(\varpi, w)= \begin{cases}1 & \mu(\varpi(\vartheta), w(\vartheta)) \geq 0, \vartheta \in \digamma \\ 0 & \text { else. }\end{cases}
$$

So for $\varpi, w \in C(\digamma)$ with $\mu(\varpi(\vartheta), w(\vartheta)) \geq 0$, we have

$$
\alpha(\varpi, w) 8 d(O \varpi, O w) \leq 8 d(O \varpi, O w) \leq \lambda(\phi(d(\varpi, w))) \phi(d(\varpi, w)), \quad \lambda \in \digamma .
$$


So, we conclude that $O$ is a $\alpha-\phi$-GC type mapping.

From (iii), we get

$$
\begin{aligned}
\alpha(\varpi, w) \geq 1 & \Rightarrow \mu(\varpi(\vartheta), w(\vartheta)) \geq 0 \quad \Rightarrow \quad \mu(O(\varpi), O(w)) \geq 0 \\
& \Rightarrow \alpha(O(\varpi), O(w)) \geq 1,
\end{aligned}
$$

for $\varpi, w \in C(\digamma)$. Thus, $O$ is $\alpha$-admissible. From (ii), there exists $\varpi_{0} \in C(\digamma)$ with $\alpha\left(\varpi_{0}, O \varpi_{0}\right) \geq 1$. By (iv) and Theorem 2.7, we find $\varpi^{*}$ with $\varpi^{*}=O \varpi^{*}$, that is, a positive solution of (4).

\section{Theorem 3.2 Suppose that}

(i) $\exists g: \digamma \times \mathbb{R}^{+} \rightarrow \mathbb{R}^{+}$such that

$$
\begin{aligned}
& |g(\vartheta, \varpi(\vartheta))-g(\vartheta, w(\vartheta))| \\
& \leq \frac{1}{2 \sqrt{2}}\left(\left(\Gamma(\iota-1)\left[\kappa(1)-\kappa(0)+\kappa^{\prime}(1)-\kappa^{\prime}(0)\right]\right)^{-1} \frac{\kappa^{\prime}(0)}{\iota-1}\right. \\
& \left.\quad \times(\kappa(1)-\kappa(0))^{\iota-1}\left(1+\frac{1}{\iota}(\kappa(1)-\kappa(0))\right)+\frac{1}{\Gamma(\iota+1)}(\kappa(1)-\kappa(0))^{\iota}\right)^{-1} \\
& \quad \times \sqrt{\phi\left(\left\|(\varpi-w)^{2}\right\|_{\infty}\right) \lambda\left(\phi\left(\left\|(\varpi-w)^{2}\right\|_{\infty}\right)\right)},
\end{aligned}
$$

where $\phi \in \Phi$ and $\lambda \in \mathcal{F}$;

(ii) $\exists \varpi_{0} \in C(\digamma)$ and $\mu: \mathbb{R}^{2} \rightarrow \mathbb{R}$ with $\mu\left(\varpi_{0}(\vartheta), \int_{0}^{1} \Lambda(\varkappa, \vartheta) \kappa^{\prime}(\vartheta) g\left(\vartheta, \varpi_{0}(\vartheta)\right) d \vartheta\right) \geq 0$, $\vartheta \in \digamma$;

(iii) for $\vartheta \in \digamma$ and $\varpi, w \in C(\digamma), \mu(\varpi(\vartheta), w(\vartheta)) \geq 0$ implies

$$
\mu\left(\int_{0}^{1} \Lambda(\varkappa, \vartheta) \kappa^{\prime}(\vartheta) g(\vartheta, \varpi(\vartheta)) d \vartheta, \int_{0}^{1} \Lambda(\varkappa, \vartheta) \kappa^{\prime}(\vartheta) g(\vartheta, w(\vartheta)) d \vartheta\right) \geq 0
$$

(iv) if $\left\{\varpi_{n}\right\} \subseteq C(\digamma)$ with $\varpi_{n} \rightarrow \varpi$ and $\mu\left(\varpi_{n}, \varpi_{n+1}\right) \geq 0$, then $\mu\left(\varpi_{n}, \varpi\right) \geq 0$.

Then (6) has at least one solution.

Proof By Lemma 2.10, $\varpi \in C(\digamma)$ is a solution of (6) if and only if $\varpi$ is a solution of the integral equation $\varpi(\varkappa)=\int_{0}^{1} \Lambda(\varkappa, \vartheta) \kappa^{\prime}(\vartheta) g(\vartheta, \varpi(\vartheta)) d \vartheta, \varkappa \in \digamma$. Define $O: C(\digamma) \rightarrow C(\digamma)$ by $O \varpi(\varkappa)=\int_{0}^{1} \Lambda(\varkappa, \vartheta) \kappa^{\prime}(\vartheta) g(\vartheta, \varpi(\vartheta)) d \vartheta$. Let $\varpi, w \in C(\digamma)$ be such that $\mu(\varpi(\varkappa), w(\varkappa)) \geq 0$. On the one hand we have

$$
\begin{aligned}
\mid O \varpi(\varkappa)-O w(\varkappa))\left.\right|^{2} \\
\quad=\left|\int_{0}^{1} \Lambda(\varkappa, \vartheta) \kappa^{\prime}(\vartheta) g(\vartheta, \varpi(\vartheta)) d \vartheta-\int_{0}^{1} \Lambda(\varkappa, \vartheta) \kappa^{\prime}(\vartheta) g(\vartheta, w(\vartheta)) d \vartheta\right|^{2} \\
\quad \leq\left[\int_{0}^{1} \Lambda(\varkappa, \vartheta) \kappa^{\prime}(\vartheta)|g(\vartheta, \varpi(\vartheta))-g(\vartheta, w(\vartheta))| d \vartheta\right]^{2} .
\end{aligned}
$$

By Lemma 2.10, for $0<\vartheta<\varkappa<1$ we have

$$
\int_{0}^{1} \Lambda(\varkappa, \vartheta) \kappa^{\prime}(\vartheta) d \vartheta
$$




$$
\begin{aligned}
\leq & \left(\Gamma(\iota-1)\left[\kappa(1)-\kappa(0)+\kappa^{\prime}(1)-\kappa^{\prime}(0)\right]\right)^{-1}\left(\kappa^{\prime}(0)+\kappa(0)-\kappa(\varkappa)\right) \\
& \times \int_{0}^{1}\left[(\kappa(1)-\kappa(\vartheta))^{\iota-2}+\frac{1}{\iota-1}(\kappa(1)-\kappa(\vartheta))^{\iota-1}\right] \kappa^{\prime}(\vartheta) d \vartheta \\
& +\frac{1}{\Gamma(\iota)} \int_{0}^{1}(\kappa(1)-\kappa(\vartheta))^{\iota-1} \kappa^{\prime}(\vartheta) d \vartheta \\
\leq & \left(\Gamma(\iota-1)\left[\kappa(1)-\kappa(0)+\kappa^{\prime}(1)-\kappa^{\prime}(0)\right]\right)^{-1} \frac{\kappa^{\prime}(0)}{\iota-1} \\
& \left.\times\left\{-[\kappa(1)-\kappa(\vartheta))^{\iota-1}\right]_{0}^{1}+\frac{1}{\iota}\left[-(\kappa(1)-\kappa(\vartheta))^{\iota}\right]_{0}^{1}\right\} \\
& +\frac{1}{\Gamma(\iota+1)}\left[-(\kappa(1)-\kappa(\vartheta))^{\iota}\right]_{0}^{1} \\
\leq & \left(\Gamma(\iota-1)\left[\kappa(1)-\kappa(0)+\kappa^{\prime}(1)-\kappa^{\prime}(0)\right]\right)^{-1} \frac{\kappa^{\prime}(0)}{\iota-1} \\
& \times(\kappa(1)-\kappa(0))^{\iota-1}\left(1+\frac{1}{\iota}(\kappa(1)-\kappa(0))\right)+\frac{1}{\Gamma(\iota+1)}(\kappa(1)-\kappa(0))^{\iota} .
\end{aligned}
$$

For $0<\varkappa<\vartheta<1$, we obtain the same estimates as analogous way to the previous one.

From (i), we get

$$
\begin{aligned}
& \int_{0}^{1} \Lambda(\varkappa, \vartheta) \kappa^{\prime}(\vartheta)|g(\vartheta, \varpi(\vartheta))-g(\vartheta, w(\vartheta))| d \vartheta \\
& \leq {\left[\left(\Gamma(\iota-1)\left[\kappa(1)-\kappa(0)+\kappa^{\prime}(1)-\kappa^{\prime}(0)\right]\right)^{-1} \frac{\kappa^{\prime}(0)}{\iota-1}\right.} \\
&\left.\times(\kappa(1)-\kappa(0))^{\iota-1}\left(1+\frac{1}{\iota}(\kappa(1)-\kappa(0))\right)+\frac{1}{\Gamma(\iota+1)}(\kappa(1)-\kappa(0))^{\iota}\right] \\
& \times|g(\vartheta, \varpi(\vartheta))-g(\vartheta, w(\vartheta))| \\
& \leq \frac{1}{2 \sqrt{2}} \sqrt{\phi\left(\left\|(\varpi-w)^{2}\right\|_{\infty}\right) \lambda\left(\phi\left(\left\|(\varpi-w)^{2}\right\|_{\infty}\right)\right)} .
\end{aligned}
$$

Thus,

$$
\mid O \varpi(\varkappa)-O w(\varkappa))\left.\right|^{2} \leq \frac{1}{8} \phi\left(\left\|(\varpi-w)^{2}\right\|_{\infty}\right) \lambda\left(\phi\left(\left\|(\varpi-w)^{2}\right\|_{\infty}\right)\right) .
$$

Put, $\alpha: C(\digamma) \times C(\digamma) \rightarrow \mathbb{R}^{+}$by

$$
\alpha(\varpi, w)= \begin{cases}1 & \mu(\varpi(\vartheta), w(\vartheta)) \geq 0, \vartheta \in \digamma \\ 0 & \text { else. }\end{cases}
$$

Hence, for $\varpi, w \in C(\digamma)$ with $\mu(\varpi(\vartheta), w(\vartheta)) \geq 0$, we have

$$
\alpha(\varpi, w) 8 d(O \varpi, O w) \leq 8 d(O \varpi, O w) \leq \lambda(\phi(d(\varpi, w))) \phi(d(\varpi, w)), \quad \lambda \in \mathcal{F} .
$$

From (iii),

$$
\alpha(\varpi, w) \geq 1 \quad \Rightarrow \quad \mu(\varpi(\vartheta), w(\vartheta)) \geq 0 \quad \Rightarrow \quad \mu(O(\varpi), O(w)) \geq 0
$$




$$
\Rightarrow \quad \alpha(O(\varpi), O(w)) \geq 1
$$

for $\varpi, w \in C(\digamma)$. Thus, $O$ is $\alpha$-admissible. From (ii), there exists $\varpi_{0} \in C(\digamma)$ with $\alpha\left(\varpi_{0}, O \varpi_{0}\right) \geq 1$. By (iv) and Theorem 2.7, we find $\varpi^{*}$ with $\varpi^{*}=O \varpi^{*}$, that is, a positive solution of the problem (6).

Setting $\phi(t)=t$ and $\lambda(t)=\frac{\cos ^{2} t}{4}$ in Theorems 3.1 and 3.2 we deduce the following corollaries.

\section{Corollary 3.3 Suppose that}

(i) $\exists g: \digamma \times \mathbb{R}^{+} \rightarrow \mathbb{R}^{+}$such that

$$
\begin{aligned}
& |g(\vartheta, \varpi(\vartheta))-g(\vartheta, w(\vartheta))| \\
& \quad \leq \frac{1}{2 \sqrt{2}} \frac{\Gamma(\iota+1)}{(\kappa(1)-\kappa(0))^{\iota}} \frac{\sqrt{\left\|(\varpi-w)^{2}\right\|_{\infty} \cos ^{2}\left\|(\varpi-w)^{2}\right\|_{\infty}}}{2} ;
\end{aligned}
$$

(ii) $\exists \varpi_{0} \in C(\digamma)$ and $\mu: \mathbb{R}^{2} \rightarrow \mathbb{R}$ with $\mu\left(\varpi_{0}(\vartheta), \int_{0}^{1} \mathcal{G}(\varkappa, \vartheta) \kappa^{\prime}(\vartheta) g\left(\vartheta, \varpi_{0}(\vartheta)\right) d \vartheta\right) \geq 0$, $\vartheta \in \digamma$;

(iii) for $\vartheta \in \digamma$ and $\varpi, w \in C(\digamma), \mu(\varpi(\vartheta), w(\vartheta)) \geq 0$ implies

$$
\mu\left(\int_{0}^{1} \mathcal{G}(\varkappa, \vartheta) \kappa^{\prime}(\vartheta) g(\vartheta, \varpi(\vartheta)) d \vartheta, \int_{0}^{1} \mathcal{G}(\varkappa, \vartheta) \kappa^{\prime}(\vartheta) g(\vartheta, w(\vartheta)) d \vartheta\right) \geq 0
$$

(iv) if $\left\{\varpi_{n}\right\} \subseteq C(\digamma)$ with $\varpi_{n} \rightarrow \varpi$ and $\mu\left(\varpi_{n}, \varpi_{n+1}\right) \geq 0$, then $\mu\left(\varpi_{n}, \varpi\right) \geq 0$.

Then (4) has at least one solution.

\section{Corollary 3.4 Suppose that}

(i) $\exists g: \digamma \times \mathbb{R}^{+} \rightarrow \mathbb{R}^{+}$such that

$$
\begin{aligned}
|g(\vartheta, \varpi(\vartheta))-g(\vartheta, w(\vartheta))| & \\
\leq & \frac{1}{2 \sqrt{2}}\left(\left(\Gamma(\iota-1)\left[\kappa(1)-\kappa(0)+\kappa^{\prime}(1)-\kappa^{\prime}(0)\right]\right)^{-1} \frac{\kappa^{\prime}(0)}{\iota-1}\right. \\
& \left.\times(\kappa(1)-\kappa(0))^{\iota-1}\left(1+\frac{1}{\iota}(\kappa(1)-\kappa(0))\right)+\frac{1}{\Gamma(\iota+1)}(\kappa(1)-\kappa(0))^{\iota}\right)^{-1} \\
& \times \frac{\sqrt{\left\|(\varpi-w)^{2}\right\|_{\infty} \cos ^{2}\left\|(\varpi-w)^{2}\right\|_{\infty}}}{2},
\end{aligned}
$$

where $\phi \in \Phi$ and $\lambda \in \mathcal{F}$;

(ii) $\exists \varpi_{0} \in C(\digamma)$ and $\mu: \mathbb{R}^{2} \rightarrow \mathbb{R}$ with $\mu\left(\varpi_{0}(\vartheta), \int_{0}^{1} \Lambda(\varkappa, \vartheta) \kappa^{\prime}(\vartheta) g\left(\vartheta, \varpi_{0}(\vartheta)\right) d \vartheta\right) \geq 0$, $\vartheta \in \digamma$;

(iii) for $\vartheta \in \digamma$ and $\varpi, w \in C(\digamma), \mu(\varpi(\vartheta), w(\vartheta)) \geq 0$ implies

$$
\mu\left(\int_{0}^{1} \Lambda(\varkappa, \vartheta) \kappa^{\prime}(\vartheta) g(\vartheta, \varpi(\vartheta)) d \vartheta, \int_{0}^{1} \Lambda(\varkappa, \vartheta) \kappa^{\prime}(\vartheta) g(\vartheta, w(\vartheta)) d \vartheta\right) \geq 0
$$

(iv) if $\left\{\varpi_{n}\right\} \subseteq C(\digamma)$ with $\varpi_{n} \rightarrow \varpi$ and $\mu\left(\varpi_{n}, \varpi_{n+1}\right) \geq 0$, then $\mu\left(\varpi_{n}, \varpi\right) \geq 0$.

Then (6) has at least one solution. 


\section{Examples}

Example 4.1 Consider the $\kappa$-Ca fractional integral BVP

$$
\left\{\begin{array}{l}
{ }^{C} D_{0^{+}} \frac{3}{2}, \frac{e^{\varkappa}}{3} \varpi(\varkappa)=g(\varkappa, \varpi(\varkappa)), \quad \varkappa \in(0,1),\|\varpi\|_{\infty}<\frac{\pi}{4}, \\
\varpi(0)=\varpi(1)=0,
\end{array}\right.
$$

where $\iota=\frac{3}{2}, \kappa(\varkappa)=e^{\varkappa}$ and $g(\varkappa, \varpi(\varkappa))=\frac{3 \sqrt{\pi}}{128 \sqrt{2}} \frac{(\varkappa+3)}{\sqrt{\left(e^{\frac{1}{3}}-1\right)^{3}}} \sin \left(2\|\varpi\|_{\infty}\right)$. Let $\vartheta \in \digamma$, and $\varpi, w \in$ $\mathbb{R}$, we have

$$
\begin{aligned}
& |g(\vartheta, \varpi(\vartheta))-g(\vartheta, w(\vartheta))| \\
& \quad=\frac{3 \sqrt{\pi}}{128 \sqrt{2}} \frac{(\vartheta+3)}{\sqrt{\left(e^{\frac{1}{3}}-1\right)^{3}}}\left|\sin 2\|\varpi\|_{\infty}-\sin 2\|w\|_{\infty}\right| \\
& \quad=\frac{3 \sqrt{\pi}}{128 \sqrt{2}} \frac{(\vartheta+3)}{\sqrt{\left(e^{\frac{1}{3}}-1\right)^{3}}}\left|2 \sin \left(\|\varpi\|_{\infty}-\|w\|_{\infty}\right) \cos \left(\|\varpi\|_{\infty}+\|w\|_{\infty}\right)\right| \\
& \quad \leq \frac{3 \sqrt{\pi}}{128 \sqrt{2}} \frac{(\vartheta+3)}{\sqrt{\left(e^{\frac{1}{3}}-1\right)^{3}}}\left|2\|\varpi-w\|_{\infty} \cos \left(\|\varpi\|_{\infty}+\|w\|_{\infty}\right)\right| \\
& \quad \leq \frac{3 \sqrt{\pi}}{128 \sqrt{2}} \frac{(\vartheta+3)}{\sqrt{\left(e^{\frac{1}{3}}-1\right)^{3}}}\left|2\|\varpi-w\|_{\infty} \cos \left(\|\varpi-w\|_{\infty}\right)\right| \\
& \quad \leq \frac{3 \sqrt{\pi}}{8 \sqrt{2}} \frac{1}{\sqrt{\left(e^{\frac{1}{3}}-1\right)^{3}}} \frac{\sqrt{\left\|(\varpi-w)^{2}\right\|_{\infty} \cos ^{2}\left\|(\varpi-w)^{2}\right\|_{\infty}}}{2} \\
& \quad \leq \frac{1}{2 \sqrt{2}} \frac{\Gamma\left(\frac{5}{2}\right)}{\left(e^{\frac{1}{3}}-1\right)^{\frac{3}{2}}} \frac{\sqrt{\left\|(\varpi-w)^{2}\right\|_{\infty} \cos ^{2}\left\|(\varpi-w)^{2}\right\|_{\infty}}}{2} \\
& \quad \leq \frac{1}{2 \sqrt{2}} \frac{\Gamma\left(\frac{5}{2}\right)}{\left(e^{\frac{1}{3}}-1\right)^{\frac{3}{2}}} \frac{\sqrt{\left\|(\varpi-w)^{2}\right\|_{\infty} \cos ^{2}\left\|(\varpi-w)^{2}\right\|_{\infty}}}{2} \\
& \left.\left.\quad=\frac{1}{2 \sqrt{2}} \frac{\Gamma(\iota+1)}{(\kappa(1)-\kappa(0))^{\iota}} \sqrt{\phi\left(\left\|(\varpi-w)^{2}\right\|_{\infty}\right) \lambda\left(\phi \left(\|(\varpi-w)^{2}\right.\right.} \|_{\infty}\right)\right),
\end{aligned}
$$

where $\phi(t)=t$ and $\lambda(t)=\frac{\cos ^{2} t}{4}$ for $t \in \digamma$.

Hence, all assumptions of Corollary 3.3 hold. Therefore, (8) has a solution on $\digamma$.

Example 4.2 Consider the $\kappa$-Ca fractional integral BVP

$$
\left\{\begin{array}{l}
{ }^{c} D_{0^{+}}^{\frac{3}{2}, e^{\varkappa}} \varpi(\varkappa)=g(\varkappa, \varpi(\varkappa)), \quad \varkappa \in(0,1),\|\varpi\|_{\infty}<\frac{\pi}{4}, \\
\varpi(0)+\varpi^{\prime}(0)=0, \quad \varpi(1)+\varpi^{\prime}(1)=0,
\end{array}\right.
$$

where $\iota=\frac{3}{2}, \kappa(\varkappa)=e^{\varkappa}$ such that for

$$
g(\varkappa, \varpi(\varkappa))=\frac{1}{8 \sqrt{2}} \mid\left(\left(\sqrt{\pi}\left[e^{\varkappa}+e-1\right]\right)^{-1} 2\left(e^{\varkappa}\right)^{\frac{1}{2}}\left(1+e^{\varkappa}\right)\right.
$$




$$
\left.+2\left[e^{\varkappa}+e-1\right]_{3}^{2}\left(e^{\varkappa}\right)^{\frac{3}{2}}\right)^{-1} \mid \sin \left(2\|\varpi\|_{\infty}\right)
$$

Let $\vartheta \in \digamma$, and $\varpi, w \in \mathbb{R}$, we have

$$
\begin{aligned}
\mid g(\vartheta, & \varpi(\vartheta))-g(\vartheta, w(\vartheta)) \mid \\
\leq & \frac{1}{8 \sqrt{2}} \mid\left(\left(\sqrt{\pi}\left[e^{\vartheta}+e-1\right]\right)^{-1} 2\left(e^{\vartheta}\right)^{\frac{1}{2}}\left(1+e^{\vartheta}\right)\right. \\
& \left.+2\left(e^{\vartheta}+e-1\right) \frac{2}{3}\left(e^{\vartheta}\right)^{\frac{3}{2}}\right)^{-1}|| \sin 2\|\varpi\|_{\infty}-\sin 2\|w\|_{\infty} \mid \\
= & \frac{1}{8 \sqrt{2}} \mid\left(\left(\sqrt{\pi}\left[e^{\vartheta}+e-1\right]\right)^{-1} 2\left(e^{\vartheta}\right)^{\frac{1}{2}}\left(1+e^{\vartheta}\right)\right. \\
& \left.+2\left(e^{\vartheta}+e-1\right) \frac{2}{3}\left(e^{\vartheta}\right)^{\frac{3}{2}}\right)^{-1}|| 2 \sin \left(\|\varpi\|_{\infty}-\|w\|_{\infty}\right) \cos \left(\|\varpi\|_{\infty}+\|w\|_{\infty}\right) \mid \\
\leq & \frac{1}{8 \sqrt{2}} \mid\left(\left(\sqrt{\pi}\left[e^{\vartheta}+e-1\right]\right)^{-1} 2\left(e^{\vartheta}\right)^{\frac{1}{2}}\left(1+e^{\vartheta}\right)\right. \\
& \left.+2\left(e^{\vartheta}+e-1\right) \frac{2}{3}\left(e^{\vartheta}\right)^{\frac{3}{2}}\right)^{-1}|| 2\|\varpi-w\|_{\infty} \cos \left(\|\varpi\|_{\infty}+\|w\|_{\infty}\right) \mid \\
\leq & \frac{1}{8 \sqrt{2}} \mid\left(\left(\sqrt{\pi} e^{\vartheta}+e-1\right]\right)^{-1} 2\left(e^{\vartheta}\right)^{\frac{1}{2}}\left(1+e^{\vartheta}\right) \\
& \left.+2\left(e^{\vartheta}+e-1\right) \frac{2}{3}\left(e^{\vartheta}\right)^{\frac{3}{2}}\right)^{-1} \mid \frac{\sqrt{\left\|(\varpi-w)^{2}\right\|_{\infty} \cos ^{2}\left\|(\varpi-w)^{2}\right\|_{\infty}}}{2} \\
= & \frac{1}{2 \sqrt{2}}\left(\left(\Gamma(\iota-1)\left[\kappa(1)-\kappa(0)+\kappa^{\prime}(1)-\kappa^{\prime}(0)\right]\right)^{-1} \frac{\kappa^{\prime}(0)}{\iota-1}\right. \\
& \left.\times(\kappa(1)-\kappa(0))^{\iota-1}\left(1+\frac{1}{\iota}(\kappa(1)-\kappa(0))\right)+\frac{1}{\Gamma(\iota+1)}(\kappa(1)-\kappa(0))^{\iota}\right)^{-1} \\
& \times \sqrt{\phi\left(\left\|(\varpi-w)^{2}\right\|_{\infty}\right) \lambda\left(\phi\left(\left\|(\varpi-w)^{2}\right\|_{\infty}\right)\right) .}
\end{aligned}
$$

Hence, assumptions of Corollary 3.4 hold. So (9) has a solution on $\digamma$.

Remark 4.3 One can easily see that Eqs. (8) and (9) considered in the above examples cannot be addressed via methods in the current literature. This in a certain sense confirms the superiority of the results of this paper over previous approaches.

\section{Conclusion}

In recent years and with the explosive growth of studies of derivatives of fractional order, there have appeared tremendous numbers of papers that reported their results by using the classical FDs and FP theorems. Meanwhile, interested researchers have raised the question of the possibility of introducing a different approach that covers all classical cases.

In this paper, we provided an affirmative answer to this inquiry by investigating the notion of existence of solutions for BVPs defined within $\kappa$-generalized FD and with the help of the FP technique based on $\alpha-\phi$-GC type mappings. The results reported in this paper generalize existing results in the literature. Two examples are presented as particular cases 
for our proposed theorems. It is proved that the results obtained are consistent with our theoretical findings.

We believe that the investigation of this problem in terms of a general approach will provide an effective platform for the study of BVPs via generalized FOs.

\author{
Acknowledgements \\ J. Alzabut would like to thank Prince Sultan University for funding this work through research group Nonlinear Analysis
} Methods in Applied Mathematics (NAMAM) group number RG-DES-2017-01-17.

Funding

Not applicable.

\title{
Availability of data and materials
}

Data sharing not applicable to this article as no datasets were generated or analyzed during the current study.

\section{Competing interests}

The authors declare that they have no competing interests.

\section{Authors' contributions}

All authors read and approved the final manuscript.

\section{Author details}

'Department of Mathematics, Faculty of Basic Science, University of Bonab, Bonab, Iran. ${ }^{2}$ Department of Mathematics, Hodiedah University, Al-Hodeidah, Yemen. ${ }^{3}$ Department of Mathematics and General Sciences, Prince Sultan University, Riyadh, Saudi Arabia.

\section{Publisher's Note}

Springer Nature remains neutral with regard to jurisdictional claims in published maps and institutional affiliations.

Received: 11 June 2020 Accepted: 18 October 2020 Published online: 23 October 2020

\section{References}

1. Abbas, S., Benchohra, M., Graef, J.R., Henderson, J.: Implicit Fractional Differential and Integral Equations: Existence and Stability, vol. 26. de Gruyter, Berlin (2018)

2. Abdo, M.S., Panchal, S.K., Hussien, S.H.: Fractional integro-differential equations with nonlocal conditions and $\psi$-Hilfer fractional derivative. Math. Model. Anal. 24(4), 564-584 (2019)

3. Abdo, M.S., Panchal, S.K., Saeed, A.M.: Fractional boundary value problem with $\psi$-Caputo fractional derivative. Proc. Indian Acad. Sci. Math. Sci. 129(5), 65 (2019). https://doi.org/10.1007/s12044-019-0514-8

4. Afshari, H.: Solution of fractional differential equations in quasi-b-metric and b-metric-like spaces. Adv. Differ. Equ. 2018, 285 (2018)

5. Afshari, H., Aydi, H., Karapinar, E.: On generalized $\alpha-\psi$-Geraghty contractions on b-metric spaces. Georgian Math. J. 27(1), 9-21 (2020). https://doi.org/10.1515/gmj-2017-0063

6. Afshari, H., Baleanu, D.: Applications of some fixed point theorems for fractional differential equations with Mittag-Leffler kernel. Adv. Differ. Equ. 2020, 140 (2020). https://doi.org/10.1186/s13662-020-02592-2

7. Afshari, H., Alsulami, H.H., Karapinar, E.: On the extended multivalued Geraghty type contractions. J. Nonlinear Sci. Appl. 9, 4695-4706 (2016). https://doi.org/10.22436/jnsa.009.06.108

8. Afshari, H., Aydi, H., Karapinar, E.: Existence of fixed points of set-valued mappings in b-metric spaces. East Asian Math. J. 32(3), 319-332 (2016)

9. Afshari, H., Kalantari, S., Baleanu, D.: Solution of fractional differential equations via $\alpha$ - $\phi$-Geraghty type mappings. Adv. Differ. Equ. 2018, 347 (2018)

10. Afshari, H., Kalantari, S., Karapinar, E.: Solution of fractional differential equations via coupled fixed point. Electron. J. Differ. Equ. 2015(286), 1 (2015)

11. Agrawal, O.P.: Some generalized fractional calculus operators and their applications in integral equations. Fract. Calc Appl. Anal. 15, 4 (2012)

12. Ahmad, B., Matar, M.M. EL-Salmy, O.M.: Existence of solutions and Ulam stability for Caputo type sequential fractional differential equations of order $\varrho \in(2,3)$. Int. J. Anal. Appl. 15(1), 86-101 (2017)

13. Almeida, R.: A Caputo fractional derivative of a function with respect to another function. Commun. Nonlinear Sci. Numer. Simul. 44, 460-481 (2017)

14. Almeida, R., Malinowska, A.B., Monteiro, M.T.: Fractional differential equations with a Caputo derivative with respect to a kernel function and their applications. Math. Methods Appl. Sci. 41(1), 336-352 (2018)

15. Almeida, R.: Fractional differential equations with mixed boundary conditions. Bull. Malays. Math. Sci. Soc. 42(4), 1687-1697 (2019)

16. Aydi, H., Jeli, M., Samet, B.: On positive solutions for a fractional thermostat model with a convex-concave source term via $\phi$-Caputo fractional derivative. Mediterr. J. Math. 17(1), 16 (2020)

17. Bai, Z., Lu, H.: Positive solutions for boundary value problem of nonlinear fractional differential equation. J. Math. Anal. Appl. 311, 495-505 (2005)

18. Bai, Z:: On positive solutions of a nonlocal fractional boundary value problem. Nonlinear Anal. 72, 916-924 (2010) 
19. Cabada, A., Aleksic, S., Tomovic, T.V., Dimitrijevic, S.: Existence of solutions of nonlinear and non-local fractional boundary value problems. Mediterr. J. Math. 16(5), , S.119 (2019)

20. Czerwik, S.: Contraction mappings in b-metric spaces. Acta Math. Inform. Univ. Ostrav. 1(1), 5-11 (1993)

21. Darwich, M.A., Ntouyas, S.K.: Existence results for a fractional functional differential equation of mixed type. Commun Appl. Nonlinear Anal. 15, 47-55 (2008)

22. Diethelm, K: Analysis of Fractional Differential Equations, Lecture Notes in Mathematics. Springer, Berlin (2010)

23. El-Shahed, E.M.: Positive solutions for boundary value problem of nonlinear fractional differential equation. Abstr. Appl. Anal. 2007, 10368 (2007)

24. El-Shahed, E.M.: On the existence and stability of solution of boundary value problem for fractional integro-differential equations with complex order. Filomat 32, 8 (2018)

25. Harikrishnan, S., Elsayed, E.M., Kanagarajan, K.: Existence and uniqueness results for fractional pantograph equations involving $\phi$-Hilfer fractional derivative. Dyn. Contin. Discrete Impuls. Syst. 25, 319-328 (2018)

26. Herrmann, R.: Fractional Calculus for Physicist. World Scientific, Singapore (2014)

27. Ismail, M., Saeed, U., Alzabut, J., Rehman, M.U.: Approximate solutions for fractional boundary value problems via Green-CAS method. Mathematics 7, 1164 (2019) https://doi.org/10.3390/math7121164

28. Jarad, F., Abdeljawad, T., Baleanu, D.: On the generalized fractional derivatives and their Caputo modification. J. Nonlinear Sci. Appl. 10, 2607-2619 (2017)

29. Karapinar, E., Samet, B.: Generalized $\alpha-\psi$-contractive type mappings and related fixed point theorems with applications. Abstr. Appl. Anal. 2012, Article ID 793486 (2012)

30. Katugampola, U.N.: A new approach to generalized fractional derivatives. Bull. Math. Anal. Appl. 6(4), 1-15 (2014)

31. Katugampola, U.N.: New approach to a generalized fractional integral. Appl. Math. Comput. 218(3), 860-865 (2011)

32. Kilbas, A.A., Srivastava, H.M., Trujillo, J.J.: Theory and Applications of Fractional Differential Equations. North-Holland Mathematics Studies. Elsevier, Amsterdam (2006)

33. Kiryakova, V.: Fractional Calculus and Applications. Longman, Harlow (1994)

34. Kucche, K.D., Mali, A.D., Sousa, J.V.C.: Theory of nonlinear $\psi$-Hilfer FDE (2018). arXiv:1808.01608

35. Liang, S., Zhang, J.: Positive solutions for boundary value problems of nonlinear fractional differential equation. Nonlinear Anal. 71, 5545-5550 (2009)

36. Liu, K., Wang, J., O'Regan, D.: Ulam-Hyers-Mittag-Lefler stability for $\phi$-Hilfer fractional-order delay differential equations. Adv. Differ. Equ. 2019(1), 50 (2019). https://doi.org/10.1186/s13662-019-1997-4

37. Marasi, H.R., Afshari, H., Zhai, C.B.: Some existence and uniqueness results for nonlinear fractional partial differential equations. Rocky Mt. J. Math. 47, 571-585 (2017). https://doi.org/10.1216/RMJ-2017-47-2-1

38. Obukhovskii, V., Zecca, P., Afanasova, M.: On some boundary value problems for fractional feedback control systems Differ. Equ. Dyn. Syst. (2018). https://doi.org/10.1007/s12591-018-0435-5

39. Podlubny, I.: Fractional Differential Equations. Academic Press, San Diego (1999)

40. Samet, B., Vetro, C., Vetro, P.: Fixed point theorems for $\alpha-\phi$-contractive type mappings. Nonlinear Anal. 75(4), 2154-2165 (2012). https://doi.org/10.1016/j.na.2011.10.014

41. Samko, S., Kilbas, A., Maricev, O.: Fractional Integrals and Derivatives. Gordon \& Breach, New York (1993)

42. Sousa, J.V.C., de Oliveira, E.C.: On the $\psi$-Hilfer fractional derivative. Commun. Nonlinear Sci. Numer. Simul. 60, 72-91 (2018). https://doi.org/10.1016/j.cnsns.2018.01.005

43. Vivek, D., Elsayed, E., Kanagarajan, K.: Theory and analysis of $\phi$ fractional differential equations with boundary conditions. Commun. Appl. Anal. 22, 401-414 (2018)

44. Xu, X., Jiang, D., Yuan, C.: Multiple positive solutions for the boundary value problem of a nonlinear fractional differential equation. Nonlinear Anal., Theory Methods Appl. 71(10), 4676-4688 (2009)

45. Osler, T.J.: Fractional derivatives of a composite function. SIAM J. Math. Anal. 1, 288-293 (1970)

46. Seemab, A., Rehman, M.U., Alzabut, J., Hamdi, A.: On the existence of positive solutions for generalized fractional boundary value problems. Bound. Value Probl. 2019(1), 186 (2019)

47. Wahash, H.A., Abdo, M.S., Panchal, S.K.: Existence and Ulam-Hyers stability of the implicit fractional boundary value problem with $\psi$-Caputo fractional derivative. J. Appl. Math. Comput. Mech. 19(1), 89-101 (2020). https://doi.org/10.17512/jamcm.2020.1.08

48. Zhang, S.: Positive solutions for boundary-value problems of nonlinear fractional differential equations. Electron. J Differ. Equ. 2006, 36 (2006)

\section{Submit your manuscript to a SpringerOpen ${ }^{\circ}$ journal and benefit from:}

- Convenient online submission

- Rigorous peer review

- Open access: articles freely available online

- High visibility within the field

- Retaining the copyright to your article

Submit your next manuscript at $>$ springeropen.com 